\title{
Nationale und internationale Befunde zu sexuellem Missbrauch durch katholische Geistliche
}

\author{
Sandra Fernau, Laura Treskow, Anja Stiller
}

\section{Einleitung}

Das Phänomen des sexuellen Missbrauchs an Minderjährigen durch katholische Geistliche erhielt mit dem Bekanntwerden zahlreicher Vorfälle in den Vereinigten Staaten und Irland bereits seit Mitte der 1990er Jahre weltweit größere öffentliche Aufmerksamkeit. In Deutschland geriet die Problematik erst Anfang 2010 durch die breite mediale Aufdeckung von Missbrauchsvorfällen in kirchlichen, staatlichen und privaten Einrichtungen in den Fokus des öffentlichen und wissenschaftlichen Interesses. Nachdem zunächst ehemalige Schüler des katholischen Canisius-Kollegs und der reformpädagogischen Odenwaldschule über Viktimisierungserfahrungen berichteten, folgte schließlich eine ganze Welle von Enthüllungen erlebter sexueller Gewalt in unterschiedlichen institutionellen Kontexten: Es wurden sowohl Vorfälle in öffentlichen, privaten sowie insbesondere in katholischen Bildungs- und Betreuungseinrichtungen als auch Übergriffe im Rahmen katholischer Gemeindearbeit und sonstiger kirchlich organisierter Aktivitäten bekannt.

National wie auch international lässt sich eine Forschungslücke auf dem Gebiet des sexuellen Missbrauchs in institutionellen und speziell in kirchlichen bzw. katholischen Kontexten feststellen: Zwar entstand in den vergangenen Jahrzehnten eine Vielzahl an empirischen Arbeiten zu sexueller Gewalt in familiären Umgebungen (zum Überblick: Finkelhor, 1994; Lampe, 2002). Es existieren jedoch nur wenige Studien zum sexuellen Missbrauch durch Vertreterinnen und Vertreter der katholischen Kirche. Der überwiegende Teil dieser Arbeiten stammt aus den Vereinigten Staaten, in denen seit den neunziger Jahren eine wissenschaftliche Auseinandersetzung mit der Problematik stattfand. Seit der 2010 begonnenen öffentlichen Missbrauchsdebatte entstehen vereinzelt auch Studien im deutschsprachigen Raum.

Im folgenden Kapitel werden zentrale empirische Befunde ausgewählter nationaler und internationaler Untersuchungen komprimiert dargestellt. Dabei findet eine Fokussierung auf Studien zum Phänomen des sexuellen 
Missbrauchs durch katholische Geistliche statt; außerkirchliche institutionelle Kontexte spielen lediglich eine untergeordnete Rolle - auch dann, wenn in den angeführten Untersuchungen teils verschiedene institutionelle Settings betrachtet werden. Die Aufarbeitung des Forschungsstands erhebt keinen Anspruch auf Vollständigkeit. Sie soll vielmehr einen Überblick über größer angelegte empirische Arbeiten und deren wesentliche Ergebnisse geben sowie im Anschluss daran Forschungslücken im Bereich innerkirchlicher sexueller Viktimisierung und sich hieraus ergebende Anknüpfungspunkte für die eigenen Studien skizzieren. ${ }^{1}$ Darüber hinaus wird im Rahmen dieses Beitrags keine kritische Analyse der Ergebnisse der Untersuchungen bzw. ihrer Datenquellen, Erhebungsinstrumente und Auswertungsmethoden angestrebt. Kritische Anmerkungen finden höchstens am Rande Erwähnung.

\section{Internationaler Forschungsstand}

In den Vereinigten Staaten wurden seit Beginn der 1990er Jahre Arbeiten zu sexuellem Missbrauch durch Vertreterinnen und Vertreter der katholischen Kirche veröffentlicht. Neben eher kirchenpolitisch oder theologisch orientierten Aufsätzen handelt es sich hierbei vor allem um quantitativ ausgerichtete Untersuchungen mit einer vornehmlich psychologischen Perspektive auf das Phänomen. Ein Schwerpunkt dieser Studien bildet zunächst die Auseinandersetzung mit den klerikalen Tätern und möglichen Ursachen ihres sexuell devianten Verhaltens. Im Zentrum der Betrachtung stehen hier neben verschiedenen Missbrauchstypen bzw. Tatmustern (z. B. Mercado, Tallon \& Terry, 2008) Persönlichkeitsmerkmale von Tätern, Besonderheiten in ihrer psychischen und sexuellen Entwicklung sowie mögliche sexuelle Störungen, wie beispielsweise Pädophilie oder Ephebophilie (z. B. Doyle, 2003; Haywood, Kravitz, Grossman, Wasyliw \& Hardy, 1996; Haywood, Kravitz, Wasyliw, Goldberg \& Cavanaugh, 1996; Laaser, 1991; Plante, 2003; Plante, Manuel \& Bryant, 1996; Terry et al., 2011). Grundlage dieser Untersuchungen sind - abgesehen von denen des John Jay College of Criminal Justice (Terry et al., 2011) - allerdings zumeist

1 Eine umfassendere Darlegung des nationalen und internationalen Forschungsstands zu sexueller Gewalt in unterschiedlichen Institutionen liefert die Expertise des Deutschen Jugendinstitut (DJI), in die auch Praxisliteratur für die Beratung von Betroffenen miteinbezogen wurde (Bundschuh, 2010). 
kleine, oftmals klinische Stichproben, die keine repräsentativen Aussagen über die geistlichen Täter allgemein erlauben.

Des Weiteren findet in den vergangen Jahren vermehrt eine Analyse von psychosozialen Folgen innerkirchlicher Missbrauchserfahrungen für die Betroffenen statt, häufig mit einem Fokus auf psychotraumatologischen Fragestellungen (z. B. Bottoms, Nielsen, Murray \& Filipas, 2003; Carr et al., 2010; Lueger-Schuster, 2012). Teilweise werden auch Auswirkungen der erlebten sexuellen Gewalt auf den persönlichen Glauben bzw. einzelne religiös-spirituelle Einstellungen (etwa die Beziehung zu Gott) sowie auf das Verhältnis zur katholischen Kirche betrachtet und damit die spirituelle Dimension der Traumatisierung in den Blick genommen (Benkert \& Doyle, 2009; Doyle, 2009; Rossetti, 1995). In diesem Zusammenhang setzt sich insbesondere Doyle mit dem emotionalen und psychologischen Einfluss der klerikalen Kultur, mit ihren hierarchischen Strukturen und spezifischen Glaubensvorstellungen auf die gläubigen Laien sowie die Kirchenführung (die Priester und Bischöfe) auseinander (Benkert \& Doyle, 2009; Doyle, 2003, 2006). Hierbei handelt es sich jedoch weniger um empirisch fundierte Arbeiten, sondern vielmehr um theoretisch angelegte Analysen des Phänomens aus einer kirchenkritischen Perspektive eines Experten für Kirchenrecht. Im Folgenden soll näher auf ausgewählte Studien und deren zentrale empirische Befunde eingegangen werden.

\section{II.1 Studien des John Jay College of Criminal Justice New York}

Wie eingangs erwähnt, wurden in den Vereinigten Staaten bereits in den 1990er Jahren zahlreiche Fälle sexueller Gewalt gegen Minderjährige durch katholische Geistliche bekannt. Als Reaktion auf den Missbrauchsskandal beauftragte die dortige Bischofskonferenz (United States Conference of Catholic Bishops) im Jahr 2002 das John Jay College of Criminal Justice mit der Erforschung des Problemfeldes. Ziel war eine umfassende wissenschaftliche Aufarbeitung des Phänomens innerkirchlichen sexuellen Missbrauchs. Es entstanden zwei groß angelegte Studien, die als bedeutende einschlägige Untersuchungen gelten: zum einen der „Nature and Scope Report" über die Häufigkeit sexueller Übergriffe durch katholische Priester und Diakone im Zeitraum von 1950 bis 2002 (John Jay College, 2004); zum anderen der „Causes and Context Report“, der eine soziohistorische und psychologische Analyse der Vorkommnisse innerkirchlichen sexuellen Missbrauchs vornimmt und Erklärungsansätze für mögliche Ursachen und Hintergründe der Sexualdelikte liefert (Terry et 
al., 2011). Die hier betrachtete Zeitspanne reicht von 1959 bis 2010. Da die beiden Studien zu den umfangreichsten empirischen Untersuchungen zum innerkirchlichen sexuellen Missbrauch zählen, sollen sie im vorliegenden Beitrag etwas ausführlicher dargestellt werden.

\section{„The Nature and Scope Report“}

Um einen Überblick über das Ausmaß der Missbrauchsvorfälle zu erhalten, wurden im Rahmen einer Aktenanalyse in fast allen US-amerikanischen Bistümern drei verschiedene Fragebögen eingesetzt, die je unterschiedliche Bereiche fokussieren. Die Fragebögen wurden jeweils auf Grundlage der Personalakten der beschuldigten Geistlichen von Mitarbeiterinnen und Mitarbeitern der verschiedenen Diözesen ausgefüllt; sämtliche ermittelte Informationen beziehen sich also auf die Akten der mutmaßlichen Täter (John Jay College, 2004). Anhand dieser wurden drei Bereiche untersucht:

- Die Diözese mittels eines Diözesanfragebogens, der unter anderem Angaben über die Anzahl der Kleriker, die des sexuellen Missbrauchs beschuldigt wurden, die Anzahl der Missbrauchsmeldungen sowie zur Struktur der Diözese und zum Umgang mit der Missbrauchsproblematik enthält.

- Die Gruppe der beschuldigten Geistlichen mittels eines Klerikerfragebogens, der neben soziodemografischen Daten unter anderem Angaben zu selbst gewählten Ausbildungsinhalten im Rahmen des Priesterseminars, Tätigkeitsbereichen und innerkirchlichen Funktionen, möglichen Versetzungen und dem Ausmaß wie der Art und Weise des Kontakts zu Minderjährigen enthält.

- Die Gruppe der Betroffenen mittels eines Betroffenenfragebogens, der neben soziodemografischen Daten unter anderem Angaben über die Anbahnung und Beendigung sowie die Gesamtdauer des sexuellen Missbrauchs, die Missbrauchshandlungen und die Reaktion der Verantwortlichen innerhalb der katholischen Kirche enthält.

Die Aktenanalyse liefert für den untersuchten Gesamtzeitraum (1950 bis 2002) folgende zentrale Ergebnisse (John Jay College, 2004; Terry, 2008). Insgesamt wurde gegen 4392 katholische Priester und Diakone von 10677 Betroffenen der formelle Vorwurf des sexuellen Missbrauchs er- 
hoben. Die Gesamtzahl der beschuldigten Geistlichen entspricht ca. $4 \%$ der Gesamtheit der Geistlichen, die im untersuchten Zeitraum in den Vereinigten Staaten im Amt waren. Dabei liegen gegen $44.3 \%$ der Beschuldigten mehrere Vorwürfe hinsichtlich der Ausübung sexueller Gewalt vor. Allgemein erweisen sich der Prozentsatz der mutmaßlichen Täter und die Prävalenzraten der Missbrauchsvorfälle bistumsübergreifend als vergleichbar. Kritisch anzumerken ist in diesem Zusammenhang allerdings, dass die unterschiedlichen Missbrauchskontexte und somit die institutionellen Bezüge, in denen die beschuldigten Geistlichen innerhalb der verschiedenen Diözesen tätig waren, im Rahmen der Aktenanalyse ungeklärt bleiben. Diesbezüglich wäre beispielsweise interessant, ob es eine Häufung von Missbrauchsvorkommnissen in geschlossenen Institutionen (wie Heimen und Internaten) gibt (Bundschuh, 2010).

Mit Blick auf die Betroffenen lässt sich anhand der Akten feststellen, dass ein deutlich höherer Anteil an Jungen sexuelle Übergriffe durch katholische Geistliche erlebte. So waren insgesamt $81 \%$ der Betroffenen männlich und lediglich $19 \%$ weiblich. Das Alter von knapp über der Hälfte der Betroffenen (51\%) zum Zeitpunkt des Missbrauchs betrug zwischen 11 und 14 Jahren. $27 \%$ waren 15 bis 17 Jahre alt; $22 \%$ der Betroffenen waren zehn Jahre alt oder jünger. Die Missbrauchshandlungen fanden allesamt mit Körperkontakt statt und beinhalteten unter anderem unangemessene Berührungen, Oralverkehr und (versuchte) Penetration.

Darüber hinaus untersuchte das Forscherteam des John Jay College den zeitlichen Verlauf der Missbrauchsvorfälle. Dieser ist charakterisiert durch einen Anstieg der jährlichen Fälle insbesondere ab den 1960er Jahren, mit einem Höhepunkt der Missbrauchszahlen in den 1970er Jahren. Seit Beginn der 1980er Jahre ist hingegen ein starker Rückgang der Missbrauchsvorkommnisse zu verzeichnen, und die Anzahl jährlicher Fälle sinkt bis 2002 weiter kontinuierlich. ${ }^{2}$

\section{„The Causes and Context Report“}

Im Jahr 2011 wurde ein weiterer Forschungsbericht des John Jay College veröffentlicht, der Ergebnisse des „Nature and Scope Report“ aufgreift

2 Dieser Rückgang ist laut der Analyse des John Jay College nicht auf eine Abnahme der gemeldeten, sondern vielmehr auf eine Abnahme der Missbrauchsvorkommnisse allgemein zurückzuführen. 
und diese mittels einer soziohistorischen und psychologischen Analyse der Missbrauchsvorkommnisse vertieft (Terry et al., 2011). Hierzu wurden vielfältige andere Datenquellen bzw. Datensätze in die Untersuchung miteinbezogen. Das primäre Ziel bestand darin, Erklärungsansätze für mögliche Ursachen und Hintergründe der Sexualdelikte zu liefern. Im Zentrum der Betrachtung stand dabei die Explikation des oben erwähnten zeitlichen Verlaufs der Missbrauchsvorfälle, das heißt der Zunahme eben dieser in den 1960er und 1970er Jahren und deren Rückgang seit 1980.

Neben den im Rahmen der umfassenden Aktenanalyse des „Nature and Scope Report" erhobenen Daten wurde auf folgendes Datenmaterial zurückgegriffen:

- Längsschnittanalysen von Datensätzen über verschiedene Arten des Sozialverhaltens (z. B. Verbrechen, Scheidung, vorehelicher Geschlechtsverkehr) im Zeitraum der untersuchten Missbrauchsvorfälle zur historischen Rahmung,

- Analysen der Teilnehmerzahl an Priesterseminaren sowie der Geschichte und Entwicklung von Ausbildungselementen in der Priesterausbildung,

- schriftliche Befragungen von und Interviews mit inaktiven katholischen Priestern, gegen die Anschuldigungen vorliegen, sowie mit einer Vergleichsgruppe von praktizierenden katholischen Priestern, gegen die keine Anschuldigungen vorliegen,

- schriftliche Befragungen von Betroffenen und Missbrauchsbeauftragten,

- schriftliche Befragungen von Bischöfen, Priestern und anderen Personen in klerikalen Leitungspositionen über nach 1985 eingeführte allgemeine kirchliche Leitlinien,

- Auswertungen von Daten (primär basierend auf Interviews mit katholischen Priestern) einer psychologischen Studie, die sich mit Persönlichkeitsmerkmalen und dem psychischen Entwicklungsstand von Priestern befasst (Loyola University Study, 1971; siehe hierzu: Kennedy, Heckler, Kobler \& Walker, 1977),

- Analysen klinischer Daten aus den Akten dreier Behandlungszentren, die katholische Priester mit sexuell deviantem Verhalten sowie mit psychischen Erkrankungen behandelten.

Als mögliche Erklärungen für den zeitlichen Verlauf der Missbrauchsvorfälle wurden vier Faktoren untersucht (Terry et al., 2011): Erstens der historische und soziokulturelle Kontext, zweitens die klerikale Ausbildung in 
den Priesterseminaren, zum Dritten individualpsychologische Merkmale der Geistlichen und viertens innerkirchliche organisationale Faktoren.

Mit Blick auf den soziohistorischen Kontext ergaben die Längsschnittanalysen zu Entwicklungstendenzen im Sozialverhalten einen $\mathrm{Zu}$ sammenhang zwischen der Zunahme an innerkirchlichen Missbrauchsfällen in den 1960er und 1970er Jahren und allgemeinen soziokulturellen Veränderungen in der US-amerikanischen Gesellschaft. So stimme der Anstieg missbräuchlicher Verhaltensweisen von Priestern mit der gesamtgesellschaftlichen Zunahme von anderen Formen des Verbrechens (bspw. Drogenkonsum) und mit allgemeinen Veränderungen im Sozialverhalten (etwa dem Anstieg vorehelichen Geschlechtsverkehrs, erhöhter Scheidungsraten) überein. Die Forscherinnen und Forscher gehen davon aus, dass die Zunahme der innerkirchlichen Missbrauchszahlen von diesen allgemeinen sozialen Entwicklungen beeinflusst wurde, wobei für die Anfälligkeit einzelner Priester in dieser Zeitperiode des soziokulturellen Wandels auch organisationale, individualpsychologische und situative Faktoren eine Rolle spielten.

Zum Rückgang der Vorkommnisse sexueller Gewalt ab den 1980er und insbesondere in den 1990er Jahren trugen den Autorinnen und Autoren zufolge Veränderungen in der klerikalen Ausbildung sowie im innerkirchlichen Umgang mit bekannt gewordenen Missbrauchsfällen bei. Diese Entwicklungen hängen ebenfalls mit gesellschaftlichen Veränderungsprozessen zusammen, die allgemein zu einer stärkeren Befassung mit der sexuellen Viktimisierung von Kindern und Jugendlichen führten und eine größere Sensibilität für diese Thematik nach sich zogen. Im Rahmen der Priesterseminare wurde ab den 1980er Jahren beispielsweise vermehrt auf die Wahrung eines professionellen Nähe-Distanz-Verhältnisses eingegangen. Die kirchlichen Reaktionen auf das Bekanntwerden von Missbrauchsvorfällen änderten sich vor allem seit den 1990er Jahren dahingehend, dass die Betroffenenperspektive tendenziell eine stärkere Berücksichtigung fand, während diese in den Jahrzehnten zuvor meist vernachlässigt worden war. Des Weiteren führte die US-amerikanische Bischofskonferenz 1992 allgemeine Leitlinien („Five Principles“) zum Umgang mit sexuellem Missbrauch ein. ${ }^{3}$

3 Hierzu merken die Autorinnen und Autoren allerdings an, dass keine einheitliche Umsetzung der Leitlinien in den verschiedenen Diözesen stattfand und so partiell weiterhin die Tendenz bestand, Missbrauchsvorfällen kirchenintern nach kanonischem Recht und nicht strafrechtlich nachzugehen. 
In Bezug auf individualpsychologische Merkmale von Geistlichen seien abschließend zwei Ergebnisse der Studie erwähnt, die im Gegensatz zum medial häufig postulierten Zusammenhang zwischen sexuellem Missbrauch innerhalb der katholischen Kirche und pädophilen Neigungen der geistlichen Täter einerseits, dem priesterlichen Pflichtzölibat andererseits stehen. Lediglich ein kleiner Prozentsatz der beschuldigten Priester und Diakone (weniger als $5 \%$ ) zeigte ein Sexualverhalten, das mit der Diagnose von Pädophilie ${ }^{4}$ übereinstimmt. Darüber hinaus sind diesbezüglich auch keine zeitspezifischen Besonderheiten zu erkennen: Der Prozentsatz pädophiler Geistlicher ist über die Jahrzehnte konstant geblieben und erscheint somit weitgehend unabhängig vom Verlauf der Missbrauchsvorfälle. Gleiches gilt für den priesterlichen Pflichtzölibat. Da eine zölibatäre Lebensweise durchgängig gefordert wird, erweist sich diese ebenfalls als zeitlich stabiler Faktor, der weder die $\mathrm{Zu}$ - noch die Abnahme der Missbrauchsvorfälle erklären kann. Hieraus lässt sich in Anlehnung an die Studien des John Jay College folgern, dass weder pädophile Neigungen noch der Zölibat maßgebliche Ursachen bzw. Einflussfaktoren für sexuellen Missbrauch durch katholische Geistliche darstellen.

\section{II.2 Bericht der irischen Untersuchungskommission (,Ryan-Report”)}

Wie in den Vereinigten Staaten wurden auch in Irland bereits in den 1990er Jahren zahlreiche Fälle sexuellen Missbrauchs in kirchlichen und staatlichen Institutionen öffentlich bekannt. Daraufhin gründete die irische Regierung im Mai 2000 die "Commission to Inquire into Child Abuse“ (CICA) zur Aufarbeitung der Vorkommnisse physischer und sexueller Gewalt in irischen Bildungs- und Betreuungseinrichtungen für Kinder (in kirchlicher und staatlicher Trägerschaft). Die primären Ziele der mehrjährigen Untersuchung sind neben der Analyse der historischen Entwicklung des irischen Kinderbetreuungssystems die Rekonstruktion des Ausmaßes, der Charakteristika und Auswirkungen der erlebten Gewalt in den verschiedenen institutionellen Kontexten (Ryan, 2009).

4 Pädophilie wird in der internationalen statistischen Klassifikation der Krankheiten und verwandter Gesundheitsprobleme (ICD) definiert als ausschließliche oder überwiegende ,sexuelle Präferenz für Kinder [...], die sich meist in der Vorpubertät oder in einem frühen Stadium der Pubertät befinden“ (Dilling, Mombour \& Schmidt, 2002, Kapitel V, F 65.4). 
$\mathrm{Zu}$ diesem Zwecke konnten sich in den Jahren 2000 bis 2006 Betroffene und ihre Kontaktpersonen telefonisch, schriftlich oder über eine Webseite an die Kommission wenden. Insgesamt meldeten sich 1541 Personen (inkl. Zeuginnen und Zeugen); mit 1090 von ihnen wurden Gespräche (sog. Anhörungen) durchgeführt. Von diesen allesamt selbst Betroffenen sind 592 männlich und 498 weiblich. Die Anhörungen wurden in den meisten Fällen mit Tonband aufgezeichnet. In der Regel berichteten die Betroffenen selbst und relativ frei erzählend von ihren Gewalterfahrungen. Es handelt sich also jeweils um Selbstauskünfte, die von den Forschern nicht vorstrukturiert wurden und darum eine uneinheitliche sowie teils unvollständige Datenlage mit unterschiedlichen Grundgesamtheiten bei einzelnen Fragestellungen generieren. Insgesamt wurden über 2000 Stunden Gesprächsmaterial aufgenommen. Im Durchschnitt dauerte eine Anhörung zwischen einer und zwei Stunden. Die Sammlung und Sortierung der Zeugenaussagen erfolgte in einer Datenbank, in der detaillierte Informationen zusammenfassend festgehalten und nach bestimmten Kategorien klassifiziert werden konnten. Eine wichtige Rolle spielte dabei die Differenzierung von verschiedenen Formen der erlebten Gewalt. Es werden im Rahmen der Datenauswertung vier Formen unterschieden: physische Gewalt, sexuelle Gewalt, Vernachlässigung sowie psychische Gewalt.

Insgesamt beziehen sich die geschilderten Gewalterfahrungen auf einen Zeitraum von 1914 bis 2000, wobei anhand der Nennungen insbesondere in den 1960er Jahren eine Häufung an Vorkommnissen zu verzeichnen ist. Es wurden Übergriffe in 216 Einrichtungen mit teils unterschiedlichen institutionellen Settings berichtet, darunter: Industrial and Reformatory Schools, ${ }^{5}$ Grundschulen, weiterführende Schulen, Internate, Kinderheime, Wohnheime, Pflegeunterbringungen und Krankenhäuser. Somit geraten kirchlich und staatlich geführte Einrichtungen sowie unterschiedliche Misshandlungs- bzw. Missbrauchskontexte in den Blick. Mittels dieser Differenzierung von verschiedenartigen institutionellen Bezügen ließen sich grundsätzlich Aussagen über Prävalenzraten von Vorfällen in unterschiedlichen institutionellen Settings treffen. Diese Möglichkeit schöpfen die Autoren des Ryan Reports allerdings nicht voll aus, da anstelle konkreter Prozentzahlen lediglich eher vage bleibende Umschreibungen gelie-

5 Es handelt sich hierbei um Unterbringungs- und Betreuungseinrichtungen für vernachlässigte, verlassene oder verwaiste Kinder, die in Irland von kirchlichen (vor allem katholischen) Kongregationen geleitet und von öffentlicher Hand finanziert worden sind. 
fert werden. Jene Problematik zeichnet sich auch an anderen Stellen der Berichte ab, beispielsweise bei den teils recht allgemein bleibenden Aussagen über den Zeitverlauf und die Gesamthäufigkeit der Vorfälle oder der Analyse der Reaktionen des sozialen Umfelds auf die Offenbarung der sexuellen Missbrauchserfahrungen. In der folgenden Zusammenfassung zentraler Erkenntnisse der verschiedenen Berichte deutet sich diese Problematik ebenfalls an.

Hinsichtlich der unterschiedlichen institutionellen Bezüge zeigt sich grundsätzlich eine Häufung von Gewaltvorkommnissen in geschlossenen Institutionen: Die Mehrheit der Befragten gab an, bereits in früher Kindheit in geschlossenen Betreuungseinrichtungen untergebracht worden $\mathrm{zu}$ sein, zumeist ohne Kontakt zu Familienangehörigen. Mehr als $90 \%$ aller Betroffenen berichteten von physischen Gewalterfahrungen, insbesondere von Schlägen und Tritten. Sexuelle Missbrauchserfahrungen wurden von mehr als der Hälfte aller Betroffenen geschildert. Diese umfassen sexuelle Handlungen mit und ohne Körperkontakt, einschließlich vaginaler und analer Penetration, erzwungenem Oralverkehr, sexueller Belästigung und Voyeurismus. Während körperliche Gewalt mitunter öffentlich (vor anderen Kindern und Aufsichtspersonen) ausgeübt wurde und somit sichtbar war, fanden die sexuellen Übergriffe im Verborgenen statt, was zu ihrer Geheimhaltung beitrug.

Von den insgesamt 1090 Betroffenenaussagen betreffen 791 religiöse (in erster Linie katholische) Betreuungseinrichtungen, die zu den Industrial and Reformatory Schools zählen. Daher liegt die Analyse der Vorkommnisse in jenen Institutionen im Fokus der Untersuchung. Die Auswertung der durch die Anhörungen gewonnenen Daten erfolgte hier geschlechtsbezogen, wobei unter den Befragten 413 Männer und 378 Frauen sind. Geschlechtsübergreifend gaben die Betroffenen in den meisten Fällen an, mit mehreren der vier voneinander differenzierten Gewaltformen (Vernachlässigung, psychische, physische und sexuelle Gewalt) in ihrer Zeit in der Einrichtung konfrontiert worden zu sein. So beinhalten $34 \%$ der Aussagen männlicher Betroffener alle vier Formen von Gewalt, 53 \% eine Kombination von physischer und sexueller Gewalt. Insgesamt sind $59 \%$ der befragten Männer von sexuellem Missbrauch betroffen. Bei den befragten Frauen zeichnet sich ein ähnliches Bild ab, wobei die Betroffenheit von sexuellem Missbrauch allein wie auch in Verbindung mit körperlicher Gewalt geringer ist: Hier berichteten $32 \%$ von einer Konfrontation mit allen vier Gewaltformen, $33 \%$ von einer Verknüpfung von physischer und sexueller Gewalt. Insgesamt sind $34 \%$ der weiblichen Befragten von sexuellem Missbrauch betroffen. 
Eine Offenbarung der sexuellen Missbrauchserfahrungen hat laut den Autoren im Allgemeinen negative Konsequenzen für die Betroffenen nach sich gezogen: In der Regel reagierte das soziale Umfeld bzw. das Einrichtungspersonal mit Unglauben und Gegenbeschuldigungen; oftmals führte die Aufdeckung gar zu weiteren sexuellen Übergriffen.

\section{II.3 Bericht der niederländischen Untersuchungskommission}

Im Jahr 2010 wurde im Auftrag der niederländischen Bischofskonferenz (der Conference of Bishops und der Conference of Religious in the Netherlands) eine Untersuchungskommission zur wissenschaftlichen Auseinandersetzung mit sexuellen Missbrauchsfällen in katholischen Institutionen und Gemeinden in den Niederlanden eingesetzt. Die groß angelegte Studie bezieht sich auf den Zeitraum von 1945 bis 2010 und liefert unter anderem einen Überblick über das Ausmaß der Vorfälle, zentrale Charakteristika innerkirchlichen Missbrauchs sowie die psychosozialen Folgen der Taten für die Betroffenen (Deetman et al., 2011).

In die Untersuchung wurde Datenmaterial aus drei unterschiedlichen Erhebungen miteinbezogen:

- Die primäre Datenbasis der Studie stellen die bei der Kommission eingegangenen Meldungen über sexuelle Missbrauchsvorkommnisse dar, die aus verschiedenen Quellen bezogen wurden. Zum einen konnten sich Betroffene zwischen März 2010 und Dezember 2010 postalisch oder per E-Mail selbst an die Kommission wenden. Zum anderen leiteten eine Betroffenenorganisation, zwei niederländische Zeitungen und ein Radiosender, Mitteilungen von Betroffenen mit deren Erlaubnis an die Kommission weiter. Insgesamt gingen 2026 Meldungen schriftlich ein, wobei 728 davon Angaben $\mathrm{zu}$ wesentlichen von der Kommission untersuchten Aspekten enthielten und einer tieferen Analyse unterzogen wurden. ${ }^{6}$

- Aufgrund der uneinheitlichen Datenlage und teils unvollständiger Angaben in den Selbstauskünften fand im Anschluss eine Nachuntersuchung mittels eines Fragebogens statt. Dieser wurde an ins-

6 Gründe für den Ausschluss von Meldungen aus der Untersuchung waren etwa unzureichende Informationen über den sexuellen Missbrauch, Schilderungen, die nicht die von der Kommission vorgegebenen Kriterien sexuellen Missbrauchs erfüllten oder der Umstand, dass die Mitteilung nicht vom Betroffenen selbst verfasst worden war. 
gesamt 883 Personen versandt (darunter die 728 Betroffenen, deren Selbstberichte ausgewertet werden konnten sowie weitere 155 Personen, die sich schriftlich an die Kommission gewandt hatten, deren Selbstberichte jedoch $\mathrm{zu}$ wenige Informationen enthielten und daher zunächst ausgeschlossen wurden). Die Rücklaufquote des Fragebogens betrug $67.8 \%$, womit in dieser Nachfolgeuntersuchung 505 von Betroffenen ausgefüllte Fragebögen Berücksichtigung fanden.

- Zur Generierung einer umfassenden Datenbasis, die verallgemeinerbare Aussagen zulässt und einen Vergleich zwischen inner- und außerkirchlichem Missbrauch ermöglicht, erfolgte zudem eine repräsentative Bevölkerungsbefragung. In Zusammenarbeit mit TNS NIPO (einem niederländischen Umfrageinstitut) wurden insgesamt 34234 Niederländerinnen und Niederländer im Alter von mindestens 40 Jahren $^{7}$ befragt. Die Rücklaufquote betrug $85 \%$. Aus der gesamten Stichprobe waren 173 Personen von sexuellem Missbrauch durch katholische Geistliche betroffen.

Zentrale Ergebnisse der drei verschiedenen Erhebungen in Hinblick auf Art, Umfang und Kontext des erlebten innerkirchlichen Missbrauchs sind in der nachfolgenden Tabelle dargestellt.

7 Die Altersgrenze wurde aufgrund der Annahme, dass die meisten Fälle sexuellen Missbrauchs durch katholische Geistliche in den 1950er bis 1970er Jahren vorkamen, festgelegt. 
Tabelle 1. Charakteristika des berichteten sexuellen Missbrauchs durch katholische Geistliche (eigene Zusammenstellung nach Deetman et al., 2011, S. 53f.)

\begin{tabular}{|c|c|c|c|}
\hline & $\begin{array}{r}\text { Betroffenenmeldung } \\
\text { (Selbstauskunft) } \\
N=728\end{array}$ & $\begin{array}{r}\text { Betroffenenmeldung } \\
\text { (Fragebogen) } \\
N=505\end{array}$ & $\begin{array}{r}\begin{array}{r}\text { Repräsentativ- } \\
\text { befragung }\end{array} \\
N=173 / 34234\end{array}$ \\
\hline \multicolumn{4}{|l|}{$\begin{array}{l}\text { Geschlecht der } \\
\text { Betroffenen }\end{array}$} \\
\hline Männlich & $84.9 \%$ & $84.0 \%$ & $77.5 \%$ \\
\hline Weiblich & $15.1 \%$ & $16.0 \%$ & $22.5 \%$ \\
\hline \multicolumn{4}{|l|}{$\begin{array}{l}\text { Missbrauchs- } \\
\text { häufigkeit }\end{array}$} \\
\hline Einmalig & $17.4 \%$ & $16.7 \%$ & $35.7 \%$ \\
\hline Mehrmalig & $82.6 \%$ & $83.3 \%$ & $64.3 \%$ \\
\hline \multicolumn{4}{|l|}{ Dauer } \\
\hline $\begin{array}{l}\text { Weniger als ein } \\
\text { Jahr }\end{array}$ & $42.9 \%$ & $44.1 \%$ & $63.8 \%$ \\
\hline Mehr als ein Jahr & $57.1 \%$ & $55.9 \%$ & $36.2 \%$ \\
\hline \multicolumn{4}{|l|}{$\begin{array}{l}\text { Missbrauchs- } \\
\text { kontext }\end{array}$} \\
\hline Gemeinde & $14.7 \%$ & $17.0 \%$ & $20.5 \%$ \\
\hline $\begin{array}{l}\text { Bildungseinrich- } \\
\text { tung }\end{array}$ & $66.6 \%$ & $50.9 \%$ & $45.2 \%$ \\
\hline Andere Institution & $11.4 \%$ & $4.8 \%$ & $10.0 \%$ \\
\hline Zu Hause & $5.8 \%$ & $11.1 \%$ & $10.0 \%$ \\
\hline Andere Kontexte & - & $16.2 \%$ & $14.3 \%$ \\
\hline Mehrere Kontexte & $1.5 \%$ & - & - \\
\hline
\end{tabular}

Beim Vergleich der beiden Gruppen von Betroffenen (Selbstmeldung und Befragungsteilnahme) zeigt sich, dass bei den Betroffenenmeldungen häufiger wiederholt stattfindende und sich über einen längeren Zeitraum erstreckende Missbrauchshandlungen angegeben wurden. Darüber hinaus wird deutlich, dass die Selbstmelder signifikant mehr sexuelle Übergriffe in Bildungseinrichtungen nannten, während Vorkommnisse in Gemeinden und in anderen institutionellen Settings (bspw. in Kinderheimen oder Waisenhäusern) erheblich verbreiteter in der Gruppe der Befragungsteilneh- 
mer sind. Jene beiden Formen innerkirchlichen Missbrauchs scheinen in den beiden Samples der Betroffenenmeldungen unterrepräsentiert zu sein. Die Autoren nehmen an, dass die höhere Prävalenz von Missbrauchsfällen in Bildungskontexten in den Selbstberichten mit der Fokussierung der medialen Berichterstattung auf diesen Bereich zusammenhängt.

Mit Blick auf die niederländische Gesamtbevölkerung lassen sich aus der landesweiten Befragung folgende repräsentative Befunde zum sexuellen Missbrauch durch katholische Geistliche ableiten:

- Von den Befragten waren zwischen 1 von 300 (0.3\%) und 1 von $100(0.9 \%)$ als Minderjährige mit ungewollten sexuellen Annäherungsversuchen durch insgesamt ca. 800 geistliche Täterinnen und Täter konfrontiert.

- Eine unzureichende Aufsicht bzw. Kontrolle erscheint als spezifisches Problem in katholischen Institutionen und Bildungseinrichtungen (wie Kinderheimen, Waisenhäusern, Internaten und Schulen). Gerade hier ist das Personal laut den Autoren seiner Aufsichtsfunktion nicht oder nur ungenügend nachgekommen.

- Insgesamt beträgt die Zahl der in den Niederlanden von sexuellem Missbrauch in katholischen Kontexten Betroffenen schätzungsweise zwischen 10000 und 20000 .

Die Folgen der sexuellen Übergriffe für die individuelle Entwicklung und psychische Gesundheit der Betroffenen stufen die Autoren in Übereinstimmung mit einschlägigen Befunden zu sexuellem Missbrauch allgemein (z. B. Haslbeck, 2007; Whitelock, Lamb \& Rentfrow, 2013) als schwerwiegend ein. Bis heute leiden die meisten Befragten unter ihren sexuellen Gewalterfahrungen. Ein Vergleich der beiden untersuchten Gruppen von Betroffenen zeigt, dass Personen, die sich selbst bei der Kommission gemeldet hatten, in größerem Umfang von aktuell vorhandenen psychischen Symptomen (wie Angstzuständen, Panikattacken, Albträumen, Flashbacks, depressive Verstimmungen etc.) berichteten als Teilnehmerinnen und Teilnehmer der Repräsentativbefragung (Deetman et al., 2011).

\section{II.4 Bericht der wissenschaftlichen Begleitforschung zur Unabhängigen Opferschutzkommission in Österreich}

Einen besonderen Fokus auf die psychotraumatologischen Konsequenzen der sexuellen Gewalterfahrungen für die Betroffenen legt eine Untersu- 
chung der Fakultät für Psychologie der Universität Wien. Dieses Forschungsprojekt hat die Aufarbeitung innerkirchlicher sexueller Missbrauchsvorkommnisse in Österreich durch die Unabhängige Opferschutzkommission (UOK/UOA) von August 2011 bis Oktober 2012 aus einer klinisch-psychologischen Perspektive wissenschaftlich begleitet (LuegerSchuster, 2012).

Im Rahmen der Untersuchung wurde auf unterschiedliches Datenmaterial zurückgegriffen: Zum einen erfolgte eine Auswertung der von Betroffenen zur Verfügung gestellten persönlichen Dokumente und Clearingberichte ${ }^{8}$, die neben personenbezogenen Angaben unter anderem Informationen zum Täter sowie zur Art und zum Ausmaß der Gewalterlebnisse enthielten. Diesbezüglich konnten die Daten von insgesamt 448 Betroffenen analysiert werden, wobei Männer mit $75.7 \%$ deutlich häufiger vertreten waren als Frauen (24.3\%). Zum anderen wurde eine Fragebogenuntersuchung durchgeführt, an der von jenen Personen nochmals 185 Betroffene $(76.2 \%$ waren männlich, $23.8 \%$ weiblich) teilnahmen. Wiederum 48 von diesen Betroffenen gaben den Forschern außerdem ein Tiefeninterview.

Aus der Datenanalyse der 448 Studienteilnehmerinnen und -teilnehmer ergibt sich mit Blick auf zentrale Charakteristika des sexuellen Missbrauchs durch katholische Geistliche Folgendes: Die meisten der in den Berichten angeführten Taten fanden in geschlossenen Institutionen statt: $39.6 \%$ in Heimen und $30.3 \%$ in Internaten. Kirchliche Schulen wurden in $10.1 \%$ und Pfarreien (bzw. Pfarr- oder Kirchräume) in $11.2 \%$ der Fälle als Tatorte angegeben. Insgesamt berichteten etwa zwei Drittel der teilnehmenden Personen von sexuellen Missbrauchserfahrungen in katholischen Kontexten. Dabei zeigte sich bei Jungen eine signifikant häufigere Betroffenheit von sexuellen Übergriffen durch katholische Geistliche: Während jede zweite Frau als Minderjährige mit sexueller Gewalt konfrontiert wurde, erlebten drei von vier Männern in ihrer Kindheit derartige Erfahrungen.

In der Fragebogenuntersuchung ist besonders auffällig, dass 90 der insgesamt 185 Befragten (48.9\%) nach einem vom Forscherteam eingesetzten gängigen Screeningverfahren (PCL-C) ${ }^{9}$ eine Posttraumatische Belas-

8 Unter Clearingberichten werden hier von Psychologinnen und Psychologen verfasste Protokolle über Gespräche mit Betroffenen verstanden.

9 Die PCL-C (Posttraumatic Stress Disorder Checklist - Civilian Version) ist ein Selbsteinschätzungsinstrument zur Beurteilung einer derzeitigen Posttraumatischen Belastungsstörung (PTBS). Es basiert auf dem Kriterienkatalog des Diagnostischen 
tungsstörung (PTBS) aufweisen. Hierbei finden sich ebenfalls geschlechtsspezifische Unterschiede: Es sind ca. zwei Drittel der teilnehmenden Frauen und knapp die Hälfte der Männer von einer PTBS betroffen. Unter einzelnen Symptomen der Störung (wie bspw. wiederkehrenden Erinnerungen, Alpträumen, Flashbacks oder physiologischen Reaktionen) leiden insgesamt 152 Personen und somit $82.6 \%$ der Befragten. Darüber hinaus wurden im Fragebogen Skalen eines weiteren Verfahrens verwendet, das psychische Belastungen anhand von verschiedenen körperlichen und psychischen Symptomen erfasst (BSI). ${ }^{10}$ Die von den Betroffenen vorgenommenen Selbstbeurteilungen machen deutlich, dass ein jeweils großer Anteil von ihnen zum Beispiel in den Skalen „Paranoides Denken“ (70.8 \%), „Depressivität“ (64.7\%), ,Somatisierung“ (63.0\%), „Ängstlichkeit“ (59.0\%) und „Phobische Angst (53.3\%) deutlich erhöhte Werte aufweist. Dabei zeigen Frauen unter anderem eine signifikant höhere Belastung in den Bereichen „Depressivität“, und „Paranoides Denken“ sowie im Gesamtwert.

\section{Nationaler Forschungsstand}

In Deutschland beschloss die Bundesregierung im März 2010 zur Aufarbeitung der Geschehnisse die Einrichtung des Runden Tischs „Sexueller Kindesmissbrauch in Abhängigkeits- und Machtverhältnissen in privaten und öffentlichen Einrichtungen und im familiären Bereich“. Neben einer umfassenden Aufklärung der Missbrauchsvorkommnisse bestand das Ziel insbesondere darin, durch die Vernetzung verschiedener Professionen Präventions- und Interventionsansätze sowie Unterstützungsmaßnahmen für Betroffene zu entwickeln (Bundesregierung Deutschland, 2013). Begleitend zur Arbeit des Runden Tisches setzte die Bundesregierung eine Unabhängige Beauftragte zur Aufarbeitung des sexuellen Kindesmissbrauchs (UBSKM) ein. Zu ihren Aufgaben zählte unter anderem der Auf-

und Statistischen Manuals psychischer Störungen (DSM) der Amerikanischen Psychiatrischen Vereinigung und erfragt die dort angeführten 17 Symptome einer PTBS. Sie stammen aus den Kriterienbereichen „Wiedererleben“, „Vermeidung“, „Abflachung der Reagibilität“ und „erhöhtes Arousal“ (Weathers, Litz, Herman, Huska \& Keane, 1993).

10 Es handelt sich beim BSI (Brief Symptom Inventory) um ein Selbstbeurteilungsinventar zur Erfassung psychischer Beschwerden, das insgesamt 53 Fragen enthält, die sich auf eine Vielzahl unterschiedlicher körperlicher und psychischer Symptome beziehen (Derogatis \& Melisaratos, 1983). 
bau einer Anlaufstelle, an die sich von sexuellem Kindesmissbrauch Betroffene sowie ihre Kontaktpersonen schriftlich und telefonisch wenden konnten. ${ }^{11}$ Die bei der Anlaufstelle ab April 2010 eingegangenen Anrufe und Briefe wurden im Rahmen einer wissenschaftlichen Begleitforschung, durchgeführt von einem Forscherteam der Klinik für Kinder- und Jugendpsychiatrie/-psychotherapie des Universitätsklinikum Ulm, dokumentiert und ausgewertet. Hierzu liegen zwei abschließende Berichte vor: Zum einen der Abschlussbericht der Unabhängigen Beauftragten, in dem die Inanspruchnahme der Anlaufstelle bis März 2011 dokumentiert ist (Bergmann, 2011), zum anderen der Endbericht des beauftragten Universitätsklinikum Ulm, welcher die bis August 2011 eingegangenen Meldungen erfasst (Fegert et al., 2011).

Die auf diese Weise zusammengetragenen Daten zur Verbreitung sexuellen Missbrauchs wurden durch das vom Deutschen Jugendinstitut (DJI) realisierte Forschungsprojekt ,Sexuelle Gewalt gegen Mädchen und Jungen in Institutionen“ ergänzt. Das ebenfalls von der Unabhängigen Beauftragten veranlasste Projekt umfasst drei Module: Erstens wurden Literaturexpertisen zum Forschungsstand zu sexueller Gewalt in familiären und in institutionellen Kontexten sowie zu Präventionsmaßnahmen erstellt (Bundschuh, 2010; Kindler \& Schmidt-Ndasi, 2010; Zimmermann, 2010). Zweitens erfolgte eine deutschlandweite standardisierte Institutionen-Befragung, ${ }^{12}$ an der eine repräsentative Stichprobe von Schulen, Internaten und Heimen teilnahm. Im Rahmen der Untersuchung wurde vorwiegend das Leitungspersonal der jeweiligen Einrichtung schriftlich oder telefonisch nach ihnen bekannt gewordenen Verdachtsfällen und dem institutionellen Umgang mit Missbrauchsvorkommnissen befragt. Drittens wurden elf Fokusgruppen-Diskussionen mit Fachkräften sowie fünf Experten-Interviews über Erfahrungen von Betroffenen und Sachkundigen bezüglich präventiver und intervenierender Maßnahmen durchgeführt (Helming et al., 2011). Die Auswertungen des DJI-Forschungsprojekts beziehen sich allerdings allgemein auf sexuellen Missbrauch in institutionellen und nicht

11 Das im Zuge des Missbrauchsskandals geschaffene Amt besteht bis heute. Durch den amtierenden Unabhängigen Beauftragten für Fragen des sexuellen Kindesmissbrauchs wird der begonnene Ausbau von Anlauf- und Hilfsmöglichkeiten für Betroffene sowie die Weiterentwicklung präventiver und intervenierender Maßnahmen fortgesetzt (Unabhängiger Beauftragter für Fragen des sexuellen Kindesmissbrauchs, Aufgaben; http://beauftragter-missbrauch.de/course/view.php?id=139; zuletzt abgerufen am: 10.04.2014).

12 Lediglich Schulleitungen in Bayern konnten nicht befragt werden. 
speziell in katholischen Kontexten, und auf letztere wird im vorliegenden Bericht auch nicht gesondert eingegangen. Deshalb finden die dort präsentierten Befunde im Folgenden keine Berücksichtigung.

\section{III.1 Bericht der wissenschaftlichen Begleitforschung zur Anlaufstelle der Unabhängigen Beauftragten zur Aufarbeitung des sexuellen Kindesmissbrauchs}

Die im Rahmen der oben bereits erwähnten wissenschaftlichen Begleitforschung ausgewerteten Daten basieren auf den bei der Anlaufstelle eingegangenen schriftlichen und telefonischen Angaben von Betroffenen und deren Kontaktpersonen im Zeitraum von April 2010 bis August 2011. ${ }^{13}$ Grundsätzlich konnte die Anlaufstelle von allen Betroffenen sexuellen Missbrauchs und ihren Ansprechpartnerinnen und Ansprechpartnern kontaktiert werden. Der Fokus der Untersuchung liegt also nicht auf sexuellem Missbrauch durch Vertreterinnen und Vertreter der katholischen Kirche; es geraten vielmehr unterschiedliche institutionelle (öffentliche und kirchliche) sowie auch nicht-institutionelle Missbrauchskontexte in den Blick, was Vergleichsmöglichkeiten eröffnet. Es gilt dabei jedoch zu beachten, dass es sich bei den Meldungen jeweils um Selbstauskünfte handelt, die von den Forscherinnen bzw. Forschern nicht vorstrukturiert wurden und darum eine uneinheitliche und teils unvollständige Datenlage generieren. Deshalb weichen die Grundgesamtheiten innerhalb der verschiedenen Auswertungsbereiche deutlich voneinander ab (Fegert et al., 2011).

Insgesamt wurden vom Forscherteam des Universitätsklinikum Ulm 6300 inhaltlich auswertbare Anrufe und Briefe erfasst, wobei mit $66.3 \%$ die Mehrheit hiervon von Betroffenen selbst, $16.2 \%$ von deren Kontaktpersonen stammte. Die Ergebnisdarstellung bezieht sich allerdings überwiegend auf die zusammengefassten Datensätze von Betroffenen und ihren jeweiligen Ansprechpartnerinnen und Ansprechpartnern. Dies erschwert eine Vergleichbarkeit mit den bereits angeführten Studien, die allesamt ausschließlich Berichte von Betroffenen auswerten (Deetman et al., 2011; Lueger-Schuster, 2012; Ryan, 2009).

13 Aufgrund des etwas längeren Untersuchungszeitraums sowie der ausführlicheren Auswertung der Daten werden nachstehend lediglich zentrale Erkenntnisse des Endberichts skizziert und auf eine Darstellung der (sehr ähnlichen) Ergebnisse des Abschlussberichts der Unabhängigen Beauftragten verzichtet. 
Laut der Angaben von Betroffenen und deren Kontaktpersonen $(N=3721)$ fand der sexuelle Missbrauch am häufigsten im familiären Kontext $(56.6 \%)$, am zweithäufigsten in institutionellen Kontexten (29.3\%) statt. Auf diese Subpopulation von $29.3 \%$ beschränken sich die folgenden Ausführungen. Dabei bezieht sich die Ergebnisdarstellung, sofern dies die Datenaufbereitung im Endbericht zulässt, auf die Auskünfte von Betroffenen.

Die Verteilung der von den Betroffenen und ihren Kontaktpersonen angegebenen unterschiedlichen Institutionen $(N=1095)$ macht deutlich, dass kirchliche Kontexte allgemein sowie kirchliche Bildungs- und Betreuungseinrichtungen mit knapp $60 \%$ am häufigsten im Zusammenhang mit erlebten sexuellen Übergriffen genannt werden. Über $70 \%$ dieser innerkirchlichen sexuellen Missbrauchsvorfälle fanden laut der Angaben der Studienteilnehmerinnen und -teilnehmer in katholischen Kontexten statt.

Eine von den Autorinnen und Autoren durchgeführte differenzierte Analyse zum sexuellen Missbrauch in institutionellen bzw. kirchlichen Kontexten ermöglicht einen Vergleich zwischen Vorfällen in katholischen, evangelischen sowie in nicht-kirchlichen Institutionen. Hier zeigt sich, dass das Geschlechterverhältnis in kirchlichen Kontexten deutlich voneinander abweicht: Während über sexuelle Übergriffe innerhalb der katholischen Kirche mit $69.8 \%$ deutlich mehr Männer als Frauen (30.2 \%) berichteten, schilderten in evangelischen Bereichen $45.3 \%$ der Männer und somit mehr Frauen (54.7\%) Missbrauchserlebnisse. Das Geschlechterverhältnis in der Gruppe mit Missbrauchserfahrungen in nicht-kirchlichen Institutionen weist demgegenüber erneut ein Übergewicht von betroffenen männlichen Personen auf (55.7\%). Die Art und Schwere des Missbrauchs unterscheidet sich in den drei Stichproben nicht bedeutend: In allen drei Gruppen gaben ca. zwei Drittel der Betroffenen Berührungen an Körper- oder Geschlechtsteilen an. Darüber hinaus berichteten die Studienteilnehmerinnen und -teilnehmer institutionsübergreifend in den allermeisten Fällen von mehrmals vorkommenden oder regelmäßig wiederkehrenden Übergriffen. In Hinblick auf die Angaben zum Geschlecht der Täterinnen bzw. Täter zeigt sich erwartungsgemäß in allen drei Stichproben ein überwiegender Männeranteil, der lediglich geringfügig differiert. 


\section{III.2 Untersuchungen im Auftrag der Deutschen Bischofskonferenz}

Auswertung der Daten der bundesweiten Beratungshotline der Deutschen Bischofskonferenz

Neben den im Zuge der Einrichtung der verschiedenen politischen Gremien durchgeführten Untersuchungen zu sexueller Gewalt in familiären und unterschiedlichen institutionellen Kontexten erfolgte durch die Deutsche Bischofskonferenz (DBK) eine Auseinandersetzung mit sexuellen Missbrauchsfällen speziell in katholischen Gemeinden und Einrichtungen. Die Lebensberatung des Bistums Trier initiierte in ihrem Auftrag eine bundesweite Hotline mit einer Telefon- und Internetberatung, an die sich Betroffene von März 2010 bis Dezember 2012 wenden konnten. Auch hier fand mittels standardisierter Basisbögen, die nach dem Telefonat oder anhand der schriftlichen Angaben ausgefüllt wurden, eine wissenschaftliche Dokumentation der eingegangen Meldungen statt, die den Zeitraum von März 2010 bis August 2011 erfasste. Auf diese Spanne bezieht sich die Auswertung der nachfolgend skizzierten Ergebnisse. Ähnlich wie beim zuvor dargelegten Endbericht des Universitätsklinikum Ulm ist zu beachten, dass sich die Datengrundlage aufgrund des klientenzentrierten Ansatzes einer Beratungshotline als uneinheitlich und teils unvollständig erweist. Dies impliziert unterschiedliche Grundgesamtheiten bei den einzelnen Fragestellungen (Zimmer, Lappehsen-Lengler, Weber \& Götzinger, 2014).

Die im Rahmen der Telefon- und Internetberatung erhobene Gesamtstichprobe umfasst 1824 Personen, die Angaben über sexuellen Missbrauch in verschiedenen (inner- und außerkirchlichen) Kontexten machten. Es sind darin sowohl Betroffene als auch Kontaktpersonen und sonstige Anruferinnen und Anrufer enthalten, wobei mit 63.9\% die Mehrheit der Auskünfte von Betroffenen selbst, $18.8 \%$ von deren Kontaktpersonen stammt. Insgesamt wurden von den Nutzerinnen und Nutzern der Hotline als Missbrauchskontexte am häufigsten Tatorte in katholischer Trägerschaft $(68.9 \%)$ benannt. 754 Personen gaben an, von sexuellem Missbrauch durch einen kirchlichen Täter betroffen gewesen zu sein; es bleibt jedoch unklar, bei wie vielen davon es sich um katholische Geistliche und bei wie vielen um solche anderer Konfessionen handelt. An manchen Stellen sind dem Bericht außerdem als Information lediglich die summierten Anzahlen (bspw. der Beschuldigungen) zu entnehmen, während konkrete Prozentangaben fehlen. 
In Bezug auf den Zeitverlauf, die Verteilung der Vorkommnisse sowie zentrale Charakteristika des sexuellen Missbrauchs durch katholische Geistliche - auch in Hinblick auf unterschiedliche institutionelle Kontexte - ergeben sich die nachstehenden Befunde: Die berichteten sexuellen Missbrauchsfälle in katholischen Einrichtungen und Gemeinden ereigneten sich überwiegend in den 1960er und 1970er Jahren. Ab den 1990er Jahren ist ein deutlicher Rückgang zu verzeichnen. Den größten Anteil der genannten Missbrauchskontexte in katholischer Trägerschaft (von insgesamt $68.9 \%$ ) machen Pfarreien mit $32.6 \%$, gefolgt von Ordenseinrichtungen $(23.8 \%)$ und Diözesaneinrichtungen $(12.5 \%)$ aus. Bei letzteren beiden handelt es sich in allererster Linie um katholische Internate sowie Kinder- und Jugendheime. Insgesamt kommen katholische Bildungs- und Betreuungseinrichtungen also auf $36.3 \%$, womit sich in der hier vorliegenden Stichprobe ebenfalls Missbrauchsvorkommnisse in geschlossenen Institutionen häufen.

In der Datenauswertung wurde ferner deutlich, dass sich die drei Institutionstypen mit ihren andersgearteten Rahmenbedingungen und Strukturmerkmalen teils auch in Hinblick auf die sexuellen Missbrauchsvorkommnisse voneinander unterscheiden. Auffällige Differenzen finden sich beim Geschlechterverhältnis der Betroffenen sowie beim Geschlecht der Täterinnen bzw. Täter: Da es in Pfarreien verschiedene Tatorte gibt (z. B. Pfarrwohnung, Sakristei, aber auch Religionsunterricht, Ferienfreizeiten etc.) schwankt das Alter und Geschlecht der Betroffenen in diesem Missbrauchskontext am meisten. Insgesamt meldeten sich im Kontext der Pfarreien 55.2\% männliche Betroffene, womit der Anteil weiblicher Betroffener $(44.8 \%)$ in diesem Bereich mit Abstand am höchsten ist. In katholischen Internaten $(81.3 \%)$ sowie in katholischen Kinder- und Jugendheimen $(74.2 \%)$ waren demnach im Vergleich deutlich mehr Jungen von sexuellem Missbrauch betroffen. Kinder- und Jugendheime wiederum stellen mit 39.8 \% Fällen, in denen weibliche Personen als Täterinnen beschuldigt wurden, den Bereich mit dem höchsten Anteil weiblicher Beschuldigter dar. Dieser beträgt im Kontext der Pfarreien lediglich $2 \%$; und auch in den katholischen Internaten wurden zu 92.7 \% Männer als Täter angegeben.

\section{Analyse forensischer Gutachten von katholischen Geistlichen}

Ferner führte ein Forscherteam, bestehend aus führenden deutschen Psychiatern, im Auftrag der deutschen Bischofskonferenz eine Analyse der 
forensischen Gutachten katholischer Geistlicher aus den Berichtsjahren 2000 bis 2010 durch (Leygraf, König, Kröber \& Pfäfflin, 2012). Die Auswertung bezog sich auf insgesamt 78 Gutachten von katholischen Geistlichen, ${ }^{14}$ die sexueller Übergriffe oder des Besitzes bzw. Konsums kinderpornografischen Materials verdächtigt wurden. Von den insgesamt 27 deutschen (Erz-)Bistümern nahmen 21 an der Studie teil. Auf Basis einer qualitativen Gutachtenanalyse sollten neben forensisch-kriminologischen und biografischen Merkmalen vor allem Besonderheiten in der persönlichen und sexuellen Entwicklung sowie klinische Auffälligkeiten der beschuldigten Geistlichen untersucht werden. Ferner ging es um die Erfassung allgemeiner Charakteristika der sexuellen Übergriffe. In Hinblick auf ihre Befunde weisen die Autoren darauf hin, dass es sich bei der vorliegenden Stichprobe um eine selektierte Stichprobe der zwischen 2000 und 2010 aufgrund vorgeworfenen sexuellen Fehlverhaltens begutachteten katholischen Geistlichen (und nicht um eine Zufallsstichprobe aus der Grundgesamtheit aller katholischen Geistlichen) handelt. Dementsprechend seien keine generalisierenden inferenzstatistischen Schlussfolgerungen möglich (Leygraf et al., 2012).

Von den 78 analysierten Gutachten beinhalten 66 Vorwürfe sexuellen Missbrauchs; in den restlichen 12 beschränkt sich der Verdacht ausschließlich auf den Besitz und Konsum kinderpornografischen Materials. Den insgesamt 66 des sexuellen Missbrauchs beschuldigten katholischen Geistlichen wurden 576 einzelne sexuelle Übergriffe an 265 Personen vorgeworfen. Wie in den anderen angeführten Studien zeigt sich auch hier eine deutlich häufigere Betroffenheit von Jungen: Der Verdacht bezieht sich zu $75 \%(n=200)$ auf Missbrauchshandlungen an männlichen und lediglich zu $25 \%(n=65)$ auf solche an weiblichen Personen. Zum Zeitpunkt des ersten sexuellen Übergriffs war die Mehrheit der begutachteten Geistlichen als Gemeindepfarrer tätig, wobei die Straftaten vielfach zu Beginn der pastoralen Laufbahn, im Durchschnitt während der ersten 7.5 Jahre, begangen wurden. Zumeist (in $88 \%$ der Fälle) standen die Betroffenen in einem (mehr oder weniger engen) Beziehungsverhältnis zu den Beschuldigten.

Mit Blick auf klinische Auffälligkeiten der Geistlichen lässt sich Folgendes festhalten: Bei rund einem Drittel von ihnen wurde den Gutachten

14 Ausgenommen sind Ordensgeistliche, Personalreferentinnen und -referenten sowie andere Mitarbeiterinnen und Mitarbeiter der katholischen Kirche. In der vorliegenden Stichprobe finden sich also ausschließlich Täter männlichen Geschlechts. 
zufolge mindestens eine psychiatrische Diagnose gemäß der internationalen statistischen Klassifikation der Krankheiten und verwandter Gesundheitsprobleme (ICD) gestellt. Eine Pädophilie wurde bei etwa jedem zehnten Geistlichen diagnostiziert. Hervorzuheben ist diesbezüglich, dass die begutachteten Geistlichen verglichen mit anderen verurteilten Sexualstraftätern weder erhöhte Prävalenzen allgemeiner psychischer Störungen, noch einer sexuellen Präferenzstörung im Sinne einer Pädophilie aufwiesen. Diese Befunde stimmen weitestgehend mit den Ergebnissen der Untersuchung des John Jay Colleges (2004) überein - auch, wenn hier eine wesentlich kleinere und andersgeartete Stichprobe vorliegt.

\section{III.3 Bericht zur sexuellen Gewalt im Internat der Benediktinerabtei Ettal}

Abschließend soll noch eine Untersuchung Erwähnung finden, die sich durch ihren explizit qualitativ-rekonstruktiven Ansatz und ihre stärkere sozialpsychologische Perspektive von den bisher genannten unterscheidet. Es handelt sich hierbei um die Studie des Instituts für Praxisforschung und Projektberatung (IPP) über sexuelle, psychische und physische Gewalt im Internat der Benediktinerabtei Ettal. In dieser werden sowohl psychosoziale Folgen der sexuellen Übergriffe für die Betroffenen als auch organisatorisch-strukturelle Hintergründe der Missbrauchspraxis und somit unter anderem die Sozialisationsbedingungen im Klosterinternat betrachtet. Im Mittelpunkt stehen dabei einerseits die Fragen, wie es zu den Missbrauchs- und Misshandlungsvorfällen überhaupt kommen konnte und warum nicht intervenierend eingegriffen wurde. Andererseits sind mögliche Ursachen der zeitlich immens verzögerten Tatmeldungen durch die Betroffenen sowie Hintergründe der divergierenden Einschätzungen der ehemaligen Schülerinnen und Schüler in Bezug auf die von ihnen verbrachte Zeit im Internat von Interesse (Keupp, Straus, Mosser, Gmür \& Hackenschmiedt, 2013).

Die Datenbasis der Studie bilden insgesamt 41 mit ehemaligen Schülerinnen bzw. Schülern und Klosterangehörigen durchgeführte qualitative Interviews, 16 hiervon mit Betroffenen psychischer, körperlicher und / oder sexueller Gewalt, drei mit beschuldigten Geistlichen. Überdies wurde auf verschiedene, den Forschern zur Verfügung gestellte Materialien zurückgegriffen, darunter Betroffenenberichte, Briefe, E-Mails und OnlineForumsdiskussionen zu Ettal, die interne Seite der Homepage des Be- 
troffenenvereins und juristische Bewertungen durch verschiedene Rechtsanwälte. Der Untersuchungszeitraum umfasst die Jahre von 1945 bis 2000.

Mit Blick auf die psychosozialen Auswirkungen der sexuellen Übergriffe arbeiten die Autoren in zahlreichen Zitaten von Betroffenen initiale und langfristige Folgen der Taten für die individuelle Entwicklung und psychische Gesundheit der Interviewten heraus. Die im Rahmen der Interviews am häufigsten beschriebenen Folgen sind insbesondere soziale Probleme, Probleme im Bereich Beziehung und Sexualität und berufliche Schwierigkeiten. Darüber hinaus finden sich in den Schilderungen der Betroffenen die Hauptkriterien für die Diagnose einer PTBS wieder. Die von den Interviewten beschriebenen Belastungen folgen demnach einer klinisch diagnostizierbaren Dynamik, was laut der Forscher auf den traumatisierenden Charakter der Gewalterfahrungen im Internat schließen lässt.

In Bezug auf die organisatorisch-strukturellen Hintergründe der Missbrauchs- und Vertuschungspraxis nennen die Autoren vor allem die folgenden, ein derartiges Klima begünstigenden Faktoren, die im untersuchten Internat zusammenwirkten:

- der kirchlich-institutionelle Rahmen als Eigenwelt mit eigenständigem Rechts- und Handlungssystem,

- eine Haltung des ,institutionellen Narzissmus“, welche ein positives Bild der Institution nach innen wie außen pflegt und verteidigt, Fehlhandlungen und Fehlentwicklungen somit verdeckt,

- die Tabuisierung von Sexualität und der hieraus resultierende fehlende reflektierte und kontrollierte Umgang damit auf Seiten der Geistlichen,

- das monastische Lebensprinzip der Geistlichen, das zu einem Mangel an Kommunikation in der Klostergemeinschaft führte,

- der Mangel an innerkirchlicher Kommunikation in einer streng hierarchischen Organisationsform,

- der Eliteanspruch des Internats, welcher den Selektionsdruck und damit zusammenhängend das Angstniveau der Schülerinnen und Schüler sowie das Machtpotenzial der Lehrer erhöhte,

- die ungenügende pädagogische Qualifikation der Klosterangehörigen.

Zur Begründung der meist erst Jahrzehnte später erfolgten Meldungen der sexuellen Missbrauchserfahrungen durch die Betroffenen verweisen die Autoren neben wahrnehmungspsychologischen Erklärungsansätzen auf 
mehrere Aspekte, die sich aus den verschiedenen Schilderungen rekonstruieren lassen. Insbesondere auch langfristig nach den Vorfällen bestehende Schamgefühle, Ängste vor Ausgrenzung, Stigmatisierungen und negativen Reaktionen des sozialen Umfelds werden als Hintergründe des langjährigen Schweigens ausgemacht. $\mathrm{Zu}$ ähnlichen Befunden kommt auch die am Kriminologischen Forschungsinstitut (KFN) durchgeführte qualitative Interviewstudie mit Betroffenen sexuellen Missbrauchs in unterschiedlichen katholischen Kontexten (siehe Fernau, 2014; Kap. 5 in diesem Band).

\section{Zentrale Befunde, Forschungslücken und Anknüpfungspunkte}

Die Übersicht einer Auswahl von bisherigen Forschungsarbeiten zum sexuellen Missbrauch in institutionellen und insbesondere in katholischen Kontexten verfolgte das Ziel, Perspektiven und Befunde der Studien herauszuarbeiten, um generelle Charakteristika der Missbrauchsvorfälle abzuleiten. Zentrale übereinstimmende Ergebnisse der verschiedenen Studien, die einen weitestgehend kongruenten Eindruck über die Geschehnisse im nationalen und internationalen Raum erzeugen, sind die folgenden:

- Der zeitliche Verlauf der Missbrauchsvorfälle ist länderübergreifend charakterisiert durch einen Anstieg der jährlichen Fälle insbesondere ab den 1960er Jahren, mit einem Höhepunkt der Missbrauchszahlen in den 1970er Jahren. Seit den 1980er und 1990er Jahren ist hingegen ein kontinuierlicher, starker Rückgang der Missbrauchsvorkommnisse zu verzeichnen. Die meisten Vorfälle ereigneten sich demnach zwischen 1960 und 1980 (Fegert et al., 2011; John Jay College, 2004; Ryan, 2009; Terry et al., 2011; Zimmer et al., 2014).

- In mehreren Studien zeigt sich ein höherer Anteil von Missbrauchsvorkommnissen in geschlossenen Institutionen (Heimen und Internaten). Das Risiko, sexuell missbräuchlichen Handlungen durch katholische Geistliche ausgesetzt zu sein, war in diesen Einrichtungen also besonders hoch (Deetman et al., 2011; LuegerSchuster, 2012; Ryan, 2009; Zimmer et al., 2014).

- Mit Blick auf die Geschlechterverteilung der Betroffenen wurde im nationalen und internationalen Raum in allen angeführten Untersuchungen deutlich, dass die wesentliche Mehrheit der Betroffenen 
sexuellen Missbrauchs durch katholische Geistliche männlichen Geschlechts ist. Im Gegensatz zu sexuellen Übergriffen in familiären Kontexten ist im Forschungsfeld institutionellen bzw. innerkirchlichen Missbrauchs der Anteil betroffener Mädchen also deutlich geringer als der betroffener Jungen (Deetman et al., 2011; Fegert et al., 2011; John Jay College, 2004; Lueger-Schuster, 2012; Ryan, 2009; Terry et al., 2011; Zimmer et al., 2014).

- In Bezug auf das Geschlecht der Täterinnen bzw. Täter zeigt sich eine umgekehrte Verteilung: Hier ist der überwiegende Anteil männlich (Fegert et al., 2011; Zimmer et al., 2014). ${ }^{15}$ Allerdings gibt es Hinweise darauf, dass die prozentuale Geschlechtsverteilung der Beschuldigten in verschiedenen institutionellen Bezügen innerhalb des katholischen Kontextes (zwischen Heimen, Internaten und Pfarreien) variiert (Zimmer et al., 2014).

- Die verschiedenen Untersuchungen machen deutlich, dass die sexuellen, physischen und psychischen Gewalthandlungen bei der Mehrheit der Betroffenen zu diversen psychischen Belastungen führten, die sich langfristig unter anderem in den Symptomen einer PTBS zeigen (Deetman et al., 2011; Keupp et al., 2013; LuegerSchuster, 2012; Ryan, 2009).

\section{IV.1 Forschungslücken und Anknüpfungspunkte für die eigenen Studien}

Abschließend werden die im vorliegenden Sammelband untersuchten Themenfelder überblicksartig unter Bezugnahme auf die im Zuge der Literatursichtung identifizierten Forschungsdesiderate skizziert. Wesentliche Befunde der qualitativen Interviewstudie mit Betroffenen sexuellen Missbrauchs durch katholische Geistliche finden sich in Kapitel 5 des Sammelbands (Fernau, 2014). Die zentralen Ergebnisse der quantitativen Fragebogenstudie mit Betroffenen werden nach ihren inhaltlichen Schwerpunkten in den Kapiteln 4 (Hellmann, Dinkelborg, Wollinger \& Fernau, 2014), 6 (Hellmann, Dinkelborg \& Fernau, 2014) und 7 (Wollinger, Dinkelborg \& Baier, 2014) dargestellt.

15 Dazu ist anzumerken, dass in einigen Untersuchungen (bspw. denen des John Jay Colleges) nur männliche Täter (als im strengen Sinne katholische Geistliche) in die Untersuchung miteinbezogen wurden. 


\section{Quantitative Befragung}

Wie bereits erwähnt, unterliegen die zuvor angeführten Untersuchungen teils einer Vielzahl von methodischen Einschränkungen, welche die Generalisierbarkeit ihrer Ergebnisse limitieren. Beispielsweise handelt es sich bei den rekrutierten Stichproben zumeist nicht um Zufallsstichproben, sondern um „Inanspruchnahme-Populationen“ und somit um selektierte Stichproben, die nur bedingt verallgemeinerbar sind. Ferner hat diese Art der Stichprobenrekrutierung im Zusammenhang mit teilweise fehlender methodischer Kontrolle bei der Datenerhebung mitunter zu einer uneinheitlichen und für einzelne Themenbereiche unvollständigen Datenlage geführt. Gerade für den deutschen Raum fehlt hier eine Fragebogenuntersuchung, die eine einheitliche und umfassende Datenlage generieren kann. Die am KFN durchgeführte quantitative Befragung Betroffener sexuellen Missbrauchs durch katholische Geistliche setzt an diesem Desiderat an. Zwar wurde aus ökonomischen und organisatorischen Gründen ebenfalls auf eine Stichprobe selbstselektierter Betroffener zurückgegriffen, die im inferenzstatistischen Sinne keine generalisierenden Schlussfolgerungen zulässt. Durch die Konstruktion eines standardisierten Fragebogens, dessen Items teilweise bereits in vergleichbaren KFN-Studien genutzt wurden (z. B. Hellmann, 2014; Wetzels, 1997) bzw. (inter-)national standardmäßig verwendet werden (z. B. PCL-C, Teegen, 1997; BSI, Derogatis \& Melisaratos, 1983), konnte jedoch eine Vergleichbarkeit $\mathrm{zu}$ anderen Betroffenenbefragungen (z. B. Lueger-Schuster, 2012) ermöglicht werden.

Mit der Herstellung von Vergleichbarkeit werden neben der Bereitstellung standardisierter Befragungsdaten weitere Ziele verfolgt: Ein zentrales Anliegen der Befragung liegt in der Gegenüberstellung von Charakteristika und Folgen des sexuellen Missbrauchs im Allgemeinen und des sexuellen Missbrauchs durch katholische Geistliche. In der wissenschaftlichen Fachliteratur finden sich hierzu unterschiedliche Positionen. Daher sollen die im Rahmen der quantitativen Befragung gewonnenen Erkenntnisse zu Ergebnissen von zwei Repräsentativbefragungen des KFN aus den Jahren 1992 (Wetzels, 1997) und 2011 (Stadler, Bieneck \& Pfeiffer, 2012) in Beziehung gesetzt werden. Darüber hinaus wurde eine Vergleichbarkeit mit anderen Untersuchungen zum Phänomen des sexuellen Missbrauchs durch katholische Geistliche angestrebt. Zentral für diesen Vergleich waren hier vor allem die Arbeiten des John Jay Colleges (2004; Terry et al., 2011) und die österreichische Studie zum sexuellen Missbrauch innerhalb der katholischen Kirche (Lueger-Schuster, 2012). Daher wurde bei der Er- 
stellung des hier verwendeten Fragebogens ein in Teilen paralleler Aufbau zum Befragungsmaterial von Lueger-Schuster (2012) angestrebt.

Neben dem Kriterium der Vergleichbarkeit hat die quantitative Betroffenenbefragung zum Ziel, Besonderheiten sexuellen Missbrauchs durch katholische Geistliche in Deutschland nachzuzeichnen. Da standardisierte Fragen im geschlossenen Antwortformat den individuellen Leidensgeschichten der Betroffenen nicht immer gerecht werden können, beinhaltet das Befragungsmaterial spezifisch zu jedem Themenkomplex die Möglichkeit, eigene Antworten in einem offenen Format abzugeben. $\mathrm{Zu}$ dem konnten die Studienteilnehmerinnen und -teilnehmer bei Bedarf am Ende des Fragebogens ausführliche Selbstberichte des Erlebten ergänzen (siehe Fernau \& Hellmann, 2014; Kap. 3 in diesem Band). Zur Analyse von Spezifika des sexuellen Missbrauchs durch katholische Geistliche werden, wie in einigen der oben angeführten Untersuchungen auch, zunächst zentrale Charakteristika der Taten (z. B. Missbrauchskontexte, Dauer, Schwere, Geschlechtsverteilung etc.) sowie kurz- und langfristige Viktimisierungsfolgen aufgezeigt. Darüber hinaus bildet die Aufarbeitung des Umgangs der Behörden und der katholischen Kirche mit den Betroffenen einen Schwerpunkt der Auswertung. Hierbei sollen insbesondere die Reaktionen von Kirchenvertreterinnen und -vertretern auf die Offenbarung von Vorfällen sexuellen Missbrauchs aus Sicht der Betroffenen untersucht werden, um eine möglicherweise bestehende Vertuschungsproblematik aufdecken zu können. Diesbezüglich fehlen vor allem im deutschsprachigen Raum empirische Befunde. Gleiches gilt für die systematische Untersuchung von Auswirkungen der erlebten sexuellen Gewalt auf die Religiosität der Betroffenen und ihr Verhältnis zur katholischen Kirche. Hierzu existieren auch international kaum empirisch fundierte Arbeiten, sondern vorwiegend vereinzelte theoretisch angelegte Auseinandersetzungen. Die Fragebogenstudie greift diese Forschungslücke auf und betrachtet die Auswirkungen der sexuellen Missbrauchserfahrungen auf den persönlichen Glauben sowie auf Einstellungen zu Religion und Kirche.

\section{Qualitative Interviewstudie}

Wie der Studienüberblick zeigte, gibt es über die skizzierten thematischen Forschungslücken hinaus bislang außerdem kaum Untersuchungen, die sich an einem qualitativen Forschungsparadigma orientieren und sich aus einer sozialwissenschaftlichen Perspektive dem Phänomen des sexuellen 
Missbrauchs durch katholische Geistliche nähern. Allerdings können gerade durch einen rekonstruktiven, biografischen Zugang zum Forschungsfeld verbunden mit einer soziologischen Ausrichtung vertiefende Erkenntnisse über Besonderheiten von Viktimisierungserfahrungen in katholischen Kontexten und deren Bewältigung generiert werden. Ein solches Vorgehen ermöglicht zum einen die eingehende Untersuchung individueller biografischer Konstellationen, subjektiver Deutungs- und Verarbeitungsmuster sowie spezifischer Dynamiken des Geschehens. Zum anderen finden neben individuellen fallübergreifende, verallgemeinerbare Erziehungs- und Sozialisationserfahrungen in religiösen Milieus Berücksichtigung. Damit geraten auch institutionell-strukturelle Hintergründe, Bedingungen und Charakteristika religiöser Sozialisation sowie deren Einfluss auf die Betroffenen und ihren Umgang mit den sexuellen Viktimisierungserfahrungen in den Blick.

Die am KFN durchgeführte qualitative Interviewstudie mit Betroffenen sexuellen Missbrauchs setzt an diesen Forschungsperspektiven an. Im Fokus der im vorliegenden Sammelband präsentierten Befunde stehen verschiedene biografische Deutungs- und Verarbeitungsversuche der sexuellen Missbrauchserfahrungen. Dabei wird insbesondere der Rückgriff auf katholische Glaubensvorstellungen in der Auseinandersetzung mit der erlebten sexuellen Gewalt betrachtet und anhand dessen die Einwirkung einer religiösen Sozialisation auf die Betroffenen und ihren Umgang mit dem sexuellen Missbrauch beleuchtet (siehe hierzu auch Fernau, 2013). Dies eröffnet vertiefende Erkenntnisse über biografische Umgangsformen und Bewältigungsmuster innerkirchlicher Viktimisierungserfahrungen, aber auch über Wirkweisen von religiöser Sozialisation und Religiosität. In diesem Zusammenhang geraten auf einer anderen, teils indirekteren Ebene als bei der KFN-Fragebogenuntersuchung ebenso Auswirkungen der erlebten sexuellen Gewalt auf die Religiosität der Betroffenen und ihr Verhältnis zur katholischen Kirche in den Blick und werden einer tiefergehenden Analyse zugänglich.

\section{Literatur}

Benkert, M. \& Doyle, T. P. (2009). Clericalism, religious duress and its psychological impact on victims of clergy sexual abuse. Pastoral Psychology, 58, 223-238.

Bergmann, C. (2011). Abschlussbericht der Unabhängigen Beauftragten zur Aufarbeitung des sexuellen Kindermissbrauchs, Dr. Christine Bergmann. Berlin: Geschäftsstelle der Unabhängigen Beauftragten zur Aufarbeitung des sexuellen Kindesmissbrauchs. 
Bottoms, B. L., Nielsen, M., Murray, R. \& Filipas, H. (2003). Religion-related child physical abuse: Characteristics and psychological outcomes. Journal of Aggression, Maltreatment and Trauma, 8, 87-114.

Bundesregierung Deutschland. (2013). Runder Tisch - Sexueller Kindesmissbrauch. Verfügbar unter: http://www.rundertisch-kindesmissbrauch.de/index.htm

Bundschuh, C. (2010). Sexualisierte Gewalt gegen Kinder in Institutionen. Nationaler und internationaler Forschungsstand. München: Deutsches Jugendinstitut.

Carr, A., Dooley, B., Fitzpatrick, M., Flanagan, E., Flanagan-Howard, R., Tierney, K., ... \& Egan, J. (2010). Adult adjustment of survivors of institutional child abuse in Ireland. Child Abuse and Neglect, 34, 477-489.

Deetman, W., Draijer, N., Kalbfleisch, P., Merckelbach, H. Monteiro, M. \& de Vries, G. (2011). Sexual abuse of minors in the Roman Catholic church. Verfügbar unter: http:/www.onderzoekrk.nl/eerste-onderzoek/eindrapport.html

Derogatis, L. R. \& Melisaratos, N. (1983). The Brief Symptom Inventory: An introductory report. Psychological Medicine, 13, 595-605.

Dilling, H., Mombour, W. \& Schmidt, M. H. (2002). Internationale Klassifikation psychischer Störungen. ICD-10 Kapitel V (F). Klinisch-diagnostische Leitlinien (5. Auflage). Bern: Huber.

Doyle, T. P. (2003). Roman Catholic clericalism, religious duress, and clergy sexual abuse. Pastoral Psychology, 51, 189-231.

Doyle, T. P. (2006). Clericalism: Enabler of clergy sexual abuse. Pastoral Psychology, 54, 189-213.

Fegert, J., Rassenhofer, M., Schneider, T., Seitz, A., König, L. \& Spröber, N. (2011). Endbericht der wissenschaftlichen Begleitforschung zur Anlaufstelle der Unabhängigen Beauftragten zur Aufarbeitung des sexuellen Kindesmissbrauchs. Ulm: Universitätsklinikum Ulm.

Fernau, S. (2013). „Weil ich die Widmung habe, alles als von Gott zu betrachten“ Zur religiösen Deutung sexueller Missbrauchserfahrungen im kirchlichen Kontext. Psychoanalyse - Texte zur Sozialforschung, 17, 17-30.

Finkelhor, D. (1994). International epidemiology of child sexual abuse. Child Abuse and Neglect, 18, 409-417.

Haslbeck, B. (2007). Sexueller Missbrauch und Religiosität: Wenn Frauen das Schweigen brechen. Münster: LIT Verlag.

Haywood, T. W., Kravitz, H. M., Grossman, L. S., Wasyliw, O. E. \& Hardy, D. W. (1996). Psychological aspects of sexual functioning among cleric and noncleric alleged sex offenders. Child Abuse and Neglect, 20, 527-536.

Haywood, T. W., Kravitz, H. M., Wasyliw, O. E., Goldberg, J. \& Cavanaugh, J. L. (1996). Cycle of abuse and psychopathology in cleric and noncleric molesters of children and adolescents. Child Abuse and Neglect, 20, 1233-1243.

Hellmann, D. F. (2014). Repräsentativbefragung zu Viktimisierungserfahrungen in Deutschland-Forschungsbericht Nr. 122. Hannover: KFN. 
Helming, E., Kindler, H., Langmeyer, A., Mayer, M., Entleitner, C., Mosser, P. \& Wolff, M. (2011). Sexuelle Gewalt gegen Mädchen und Jungen in Institutionen Rohdatenbericht. München: Deutsches Jugendinstitut.

John Jay College (2004). The nature and scope of sexual abuse of minors by Catholic priests and deacons in the United States, 1950-2002. Washington, DC: United States Conference of Catholic Bishops (USCCB).

Kennedy, E. C., Heckler, V. J., Kobler, F. J. \& Walker, R. E. (1977). Clinical assessment of a profession: Roman Catholic clergymen. Journal of Clinical Psychology, 33, 120-128.

Keupp, H., Straus, F., Mosser, P., Gmür, W. \& Hackenschmiedt, G. (2013). Sexueller Missbrauch, psychische und körperliche Gewalt im Internat der Benediktinerabtei Ettal. München: Institut für Praxisforschung und Projektberatung.

Kindler, H. \& Schmidt-Ndasi, D. (2010). Wirksamkeit von Maßnahmen zur Prävention und Intervention im Fall sexueller Gewalt gegen Kinder. München: Deutsches Jugendinstitut.

Laaser, M. (1991). Sexual addiction and clergy. Pastoral Psychology, 39, 213-235.

Lampe, A. (2002). The prevalence of childhood sexual abuse, physical abuse and emotional neglect in Europe. Psychosomatic Medicine and Psychotherapy, 48, 370-380.

Leygraf, N., König, A., Kröber, H.-L. \& Pfäfflin, F. (2012). Sexuelle Übergriffe durch katholische Geistliche in Deutschland. Eine Analyse forensischer Gutachten 20002010. Verfügbar unter http://www.dbk.de/fileadmin/redaktion/diverse_downloads/ Dossiers_2012/2012_Sex-Uebergriffe-durch-katholische-Geistliche_LeygrafStudie.pdf

Lueger-Schuster, B. (2012). Psychotraumatologische Fragestellungen zu Gewalt und Missbrauch in der Katholischen Kirche. Wien: Universität Wien.

Mercado, C. C., Tallon, J. A. \& Terry, K. J. (2008). Persistent sexual abusers in the Catholic church: An examination of characteristics and offense patterns. Criminal Justice and Behavior, 35, 629-642.

Plante, T. G. (2003). Priests behaving badly: What do we know about priest sex offenders? Sexual Addiction and Compulsivity, 9, 93-97.

Plante, T. G., Manuel, G. \& Bryant, C. (1996). Personality and cognitive functioning among hospitalized sexual offending Roman Catholic priests. Pastoral Psychology, 45, 129-139.

Rossetti, S. J. (1995). The impact of child sexual abuse on attitudes toward God and the Catholic church. Child Abuse and Neglect, 19, 1469-1481.

Ryan, S. (2009). Report of the Commission to Inquire into Child Abuse. Dublin: Stationary Office.

Stadler, L., Bieneck, S. \& Pfeiffer, C. (2012). Repräsentativbefragung sexueller Missbrauch-Forschungsbericht Nr. 118. Hannover: KFN.

Teegen, F. (1997). Deutsche Übersetzung der Posttraumatic Stress Disorder Checklist (PCL-C) des National Center for PTSD. Hamburg: Universität Hamburg.

Terry, K. J. (2008). Stained glass: The nature and scope of child sexual abuse in the Catholic church. Criminal Justice and Behavior, 35, 549-569. 
Terry, K. J., Smith, M. L., Schuth, K., Kelly, J. R., Vollman, B. \& Massey, C. (2011). The causes and context of sexual abuse of minors by Catholic priests in the United States, 1950-2010. Washington, DC: United States Conference of Catholic Bishops (USCCB).

Weathers, F. W., Litz, B. T., Herman, D. S., Huska, J. A. \& Keane, T. M. (1993). The PTSD checklist: reliability, validity \& diagnostic utility. Paper presented at the Annual Meeting of the International Society for Traumatic Stress Sudies, San Antonio, TX.

Wetzels, P. (1997). Gewalterfahrungen in der Kindheit: Sexueller Mißbrauch, körperliche Mißhandlung und deren langfristige Konsequenzen. Baden-Baden: Nomos.

Whitelock, C. F., Lamb, M. E. \& Rentfrow, P. J. (2013). Overcoming trauma: Psychological and demographic characteristics of child sexual abuse survivors in adulthood. Clinical Psychological Science, 1, 351-362.

Zimmer, A., Lappehsen-Lengler, D., Weber, M. \& Götzinger, K. (2014). Sexueller Kindesmissbrauch in kirchlichen Institutionen - Zeugnisse, Hinweise, Prävention. Weinheim: Beltz.

Zimmermann, P. (2010). Sexuelle Gewalt gegen Kinder in Familien. München: Deutsches Jugendinstitut. 